\title{
Existence results for fractional differential equations with three-point boundary conditions
}

$\mathrm{XiFu}^{*}$

\section{"Correspondence:}

fuxi1984@hotmail.com

Department of Mathematics,

Shaoxing University, Shaoxing,

Zhejiang 312000, P.R. China

\begin{abstract}
In this paper, we study three-point boundary value problems of nonlinear fractional differential equations. Existence and uniqueness results are obtained by using standard fixed point theorems. Some examples are given to illustrate the results. MSC: 34A60; 26A33; 34B15
\end{abstract}

Keywords: fractional differential equations; boundary value problems; existence results

\section{Introduction}

Fractional differential equations have gained much importance and attention due to the fact that they have been proved to be valuable tools in the modeling of many phenomena in engineering and sciences such as physics, mechanics, economics and biology, etc. [13]. For some developments on the existence results of fractional differential equations, we can refer to [4-25] and the references therein.

In recent years, there has been a great deal of research on the questions of existence and uniqueness of solutions to boundary value problems for differential equations of fractional order. For example, Ahmad and Nieto [8] investigated the existence and uniqueness of solutions for an anti-periodic fractional boundary value problem

$$
\left\{\begin{array}{l}
{ }^{c} D^{\alpha} x(t)=f(t, x(t)), \quad t \in[0, T], 1<\alpha \leq 2, T>0, \\
x(0)=-x(T), \quad{ }^{c} D^{\gamma} x(0)=-{ }^{c} D^{\gamma} x(T), \quad 0<\gamma<1,
\end{array}\right.
$$

where ${ }^{c} D^{\alpha}$ denotes the Caputo fractional derivative of order $\alpha, f$ is a given continuous function.

In [16], the author discussed the existence of solutions for the following nonlinear fractional differential equations with anti-periodic-type fractional boundary conditions

$$
\left\{\begin{array}{l}
{ }^{c} D^{\alpha} x(t)=f\left(t, x(t),{ }^{c} D^{\beta} x(t)\right), \quad t \in[0, T], 1<\alpha \leq 2,0<\beta \leq 1, \\
x(0)+\mu_{1} x(T)=\sigma_{1}, \quad{ }^{c} D^{\gamma} x(0)+\mu_{2}{ }^{c} D^{\gamma} x(T)=\sigma_{2}, \quad 0<\gamma<1,
\end{array}\right.
$$

where ${ }^{c} D^{q}$ denotes the Caputo fractional derivative of order $q, \mu_{1} \neq-1, \mu_{1} \neq 0, \sigma_{1}, \sigma_{2}$ are real constants, and $f$ is a given continuous function.

(c) 2013 Fu; licensee Springer. This is an Open Access article distributed under the terms of the Creative Commons Attribution License (http://creativecommons.org/licenses/by/2.0), which permits unrestricted use, distribution, and reproduction in any medium, provided the original work is properly cited. 
Fractional differential equations with three-point integral boundary conditions of the following form were considered in [15] by Ahmad et al.

$$
\left\{\begin{array}{l}
{ }^{c} D^{\alpha} x(t)=f(t, x(t)), \quad t \in[0,1], 1<\alpha \leq 2, \\
x(0)=0, \quad x(1)=a \int_{0}^{\eta} x(s) d s, \quad 0<\eta<1,
\end{array}\right.
$$

where ${ }^{c} D^{\alpha}$ denotes the Caputo fractional derivative of order $\alpha, f$ is a given continuous function, and $a \in \mathbb{R}$ with $a \eta^{2} \neq 2$.

By a simple computation, we observed that ${ }^{c} D^{\gamma} x(0)=0$ in equations (1) and (2). This implies that the boundary conditions ${ }^{c} D^{\gamma} x(0)=-{ }^{c} D^{\gamma} x(T)$ in (1) and ${ }^{c} D^{\gamma} x(0)+\mu_{2}{ }^{c} D^{\gamma} x(T)=$ $\sigma_{2}$ in (2) actually are equivalent to the boundary conditions ${ }^{c} D^{\gamma} x(T)=0$ and $\mu_{2}{ }^{c} D^{\gamma} x(T)=$ $\sigma_{2}$, respectively.

Motivated by the papers above, in this article, firstly, we study fractional differential equations with the three-point boundary conditions in the following form

$$
\left\{\begin{array}{l}
{ }^{c} D^{\alpha} x(t)=f(t, x(t)), \quad t \in[0, T], 1<\alpha \leq 2, T>0, \\
a_{1} x(0)+b_{1} x(T)=c_{1}, \\
a_{2}\left({ }^{c} D^{\gamma} x(\eta)\right)+b_{2}\left({ }^{c} D^{\gamma} x(T)\right)=c_{2}, \quad 0<\eta<T, 0<\gamma<1,
\end{array}\right.
$$

where ${ }^{c} D^{q}$ denotes the Caputo fractional derivative of order $q, a_{i}, b_{i}, c_{i}, i=1,2$ are real constants such that $a_{1}+b_{1} \neq 0, a_{2} \eta^{1-\gamma}+b_{2} T^{1-\gamma} \neq 0$, and $f$ is a given continuous function.

Then we consider the fractional differential equations with three-point integral boundary conditions

$$
\left\{\begin{array}{l}
{ }^{c} D^{\alpha} x(t)=f\left(t, x(t),{ }^{c} D^{\beta} x(t)\right), \quad t \in[0,1], 1<\alpha \leq 2,0<\beta<1, \\
x(0)=0, \quad a I^{\gamma} x(\eta)+b x(1)=c, \quad 0<\eta<1,
\end{array}\right.
$$

where ${ }^{c} D^{q}$ denotes the Caputo fractional derivative of order $q, I^{\gamma}$ the Riemann-Liouville fractional integral of order $\gamma, f$ is a given continuous function, and $a, b, c$ are real constants with $a \eta^{1+\gamma} \neq-b \Gamma(\beta+2)$.

We remark that when $a_{i}=b_{i}=1, c_{i}=0$ and $\eta \rightarrow 0$, problem (3) reduces to the antiperiodic fractional boundary value problem (1) (cf. [8]).

The paper is organized as follows: in Section 2 we present the notations, definitions and give some preliminary results that we need in the sequel, Sections 3 and 4 are dedicated to the existence results of problems (4) and (5), respectively, in the final Section 5, two examples are given to illustrate the results.

\section{Preliminaries}

Definition 2.1 [17] The Riemann-Liouville fractional integral of order $q$ for a continuous function $f:[0, \infty) \rightarrow \mathbb{R}$ is defined as

$$
I^{q} f(t)=\frac{1}{\Gamma(q)} \int_{0}^{t} \frac{f(s)}{(t-s)^{1-q}} d s, \quad q>0
$$

provided the integral exists. 
Definition 2.2 [17] For $(n-1)$ times absolutely continuous function $f:[0, \infty) \rightarrow \mathbb{R}$, the Caputo derivative of fractional order $q$ is defined as

$$
{ }^{c} D^{q} f(t)=\frac{1}{\Gamma(n-q)} \int_{0}^{t}(t-s)^{n-q-1} f^{(n)}(s) d s, \quad n-1<q<n, n=[q]+1,
$$

where $[q]$ denotes the integer part of the real number $q$.

Lemma 2.1 [12] Let $\alpha>0$, then the differential equation

$$
{ }^{c} D^{\alpha} h(t)=0
$$

has solutions $h(t)=c_{0}+c_{1} t+c_{2} t^{2}+\cdots+c_{n-1} t^{n-1}$ and

$$
I^{\alpha c} D^{\alpha} h(t)=h(t)+c_{0}+c_{1} t+c_{2} t^{2}+\cdots+c_{n-1} t^{n-1},
$$

here $c_{i} \in \mathbb{R}, i=0,1,2, \ldots, n-1, n=[\alpha]+1$.

The following are two standard fixed point theorems, which will be used in Sections 3 and 4 (see [26]).

Theorem 2.1 Let $X$ be a Banach space, let $B$ be a nonempty closed convex subset of $X$. Suppose that $F: B \rightarrow B$ is a continuous compact map. Then $F$ has a fixed point in $B$.

Theorem 2.2 (Nonlinear alternative for single-valued maps) Let X be a Banach space, let $B$ be a closed, convex subset of $X$, let $U$ be an open subset of $B$ and $0 \in U$. Suppose that $P: \bar{U} \rightarrow B$ is a continuous and compact map. Then either (a) $P$ has a fixed point in $\bar{U}$, or (b) there exist an $x \in \partial U$ (the boundary of $U$ ) and $\lambda \in(0,1)$ with $x=\lambda P(x)$.

\section{Existence results for problem (4)}

Lemma 3.1 For any $y \in C([0, T], \mathbb{R})$, the unique solution of the three-point boundary value problem

$$
\left\{\begin{array}{l}
{ }^{c} D^{\alpha} x(t)=y(t), \quad t \in[0, T], 1<\alpha \leq 2, \\
a_{1} x(0)+b_{1} x(T)=c_{1}, \\
a_{2}\left({ }^{c} D^{\gamma} x(\eta)\right)+b_{2}\left({ }^{c} D^{\gamma} x(T)\right)=c_{2}, \quad 0<\eta<T, 0<\gamma<1,
\end{array}\right.
$$

is given by

$$
\begin{aligned}
x(t)= & \int_{0}^{t} \frac{(t-s)^{\alpha-1}}{\Gamma(\alpha)} y(s) d s-\frac{b_{1}}{a_{1}+b_{1}} \int_{0}^{T} \frac{(T-s)^{\alpha-1}}{\Gamma(\alpha)} y(s) d s \\
& +\frac{c_{1}}{a_{1}+b_{1}}+\frac{b_{1} T \Gamma(2-\gamma)-\left(a_{1}+b_{1}\right) \Gamma(2-\gamma) t}{\left(a_{1}+b_{1}\right)\left(a_{2} \eta^{1-\gamma}+b_{2} T^{1-\gamma}\right)} \\
& \times\left(a_{2} \int_{0}^{\eta} \frac{(\eta-s)^{\alpha-\gamma-1}}{\Gamma(\alpha-\gamma)} y(s) d s+b_{2} \int_{0}^{T} \frac{(T-s)^{\alpha-\gamma-1}}{\Gamma(\alpha-\gamma)} y(s) d s-c_{2}\right) .
\end{aligned}
$$


Proof For $1<\alpha \leq 2$, by Lemma 2.1, we know that the general solution of the equation ${ }^{c} D^{\alpha} x(t)=y(t)$ can be written as

$$
x(t)=I^{\alpha} y(t)-k_{0}-k_{1} t=\int_{0}^{t} \frac{(t-s)^{\alpha-1}}{\Gamma(\alpha)} y(s) d s-k_{0}-k_{1} t,
$$

where $k_{0}, k_{1} \in \mathbb{R}$ are arbitrary constants. Since ${ }^{c} D^{\gamma} k_{0}=0,{ }^{c} D^{\gamma} t=\frac{t^{1-\gamma}}{\Gamma(2-\gamma)},{ }^{c} D^{\gamma} I^{\alpha} y(t)=$ $I^{\alpha-\gamma} y(t)$, we have

$$
{ }^{c} D^{\gamma} x(t)=I^{\alpha-\gamma} y(t)-\frac{k_{1} t^{1-\gamma}}{\Gamma(2-\gamma)}=\int_{0}^{t} \frac{(t-s)^{\alpha-\gamma-1}}{\Gamma(\alpha-\gamma)} y(s) d s-\frac{k_{1} t^{1-\gamma}}{\Gamma(2-\gamma)} .
$$

Using the boundary conditions, we obtain

$$
\begin{aligned}
& a_{1}\left(-k_{0}\right)+b_{1}\left(\int_{0}^{T} \frac{(T-s)^{\alpha-1}}{\Gamma(\alpha)} y(s) d s-k_{0}-k_{1} T\right)=c_{1}, \\
& a_{2} \int_{0}^{\eta} \frac{(\eta-s)^{\alpha-\gamma-1}}{\Gamma(\alpha-\gamma)} y(s) d s+b_{2} \int_{0}^{T} \frac{(T-s)^{\alpha-\gamma-1}}{\Gamma(\alpha-\gamma)} y(s) d s-\frac{k_{1}\left(a_{2} \eta^{1-\gamma}+b_{2} T^{1-\gamma}\right)}{\Gamma(2-\gamma)}=c_{2} .
\end{aligned}
$$

Therefore, we have

$$
\begin{aligned}
k_{0}= & \frac{b_{1}}{a_{1}+b_{1}} \int_{0}^{T} \frac{(T-s)^{\alpha-1}}{\Gamma(\alpha)} y(s) d s-\frac{c_{1}}{a_{1}+b_{1}}-\frac{b_{1} T \Gamma(2-\gamma)}{\left(a_{1}+b_{1}\right)\left(a_{2} \eta^{1-\gamma}+b_{2} T^{1-\gamma}\right)} \\
& \times\left(a_{2} \int_{0}^{\eta} \frac{(\eta-s)^{\alpha-\gamma-1}}{\Gamma(\alpha-\gamma)} y(s) d s+b_{2} \int_{0}^{T} \frac{(T-s)^{\alpha-\gamma-1}}{\Gamma(\alpha-\gamma)} y(s) d s-c_{2}\right), \\
k_{1}= & \frac{\Gamma(2-\gamma)\left(a_{2} \int_{0}^{\eta} \frac{(\eta-s)^{\alpha-\gamma-1}}{\Gamma(\alpha-\gamma)} y(s) d s+b_{2} \int_{0}^{T} \frac{(T-s)^{\alpha-\gamma-1}}{\Gamma(\alpha-\gamma)} y(s) d s-c_{2}\right)}{a_{2} \eta^{1-\gamma}+b_{2} T^{1-\gamma}} .
\end{aligned}
$$

Substituting the values of $k_{0}, k_{1}$ in (7), we obtain the result. This completes the proof.

From the proof of Lemma 3.1, we note that when $0<\gamma<1,{ }^{c} D^{\gamma} x(\eta)=\int_{0}^{\eta} \frac{(\eta-s)^{\alpha-\gamma-1}}{\Gamma(\alpha-\gamma)} y(s) d s-$ $\frac{k_{1} \eta^{1-\gamma}}{\Gamma(2-\gamma)}$, that is to say, the non-separateness feature in (4) is more expressed than those in (1).

Let $J=[0, T]$ and $\mathcal{C}=C(J, \mathbb{R})$ be the Banach space of all continuous real functions from $J$ into $\mathbb{R}$ equipped with the norm $\|x\|=\sup _{t \in J}|x(t)|$. In view of Lemma 3.1, we define an operator $\mathcal{F}: \mathcal{C} \rightarrow \mathcal{C}$ as follows

$$
\begin{aligned}
& (\mathcal{F} x)(t) \\
& =\int_{0}^{t} \frac{(t-s)^{\alpha-1}}{\Gamma(\alpha)} f(s, x(s)) d s-\frac{b_{1}}{a_{1}+b_{1}} \int_{0}^{T} \frac{(T-s)^{\alpha-1}}{\Gamma(\alpha)} f(s, x(s)) d s \\
& \quad+\frac{b_{1} T \Gamma(2-\gamma)-\left(a_{1}+b_{1}\right) \Gamma(2-\gamma) t}{\left(a_{1}+b_{1}\right)\left(a_{2} \eta^{1-\gamma}+b_{2} T^{1-\gamma}\right)} \times\left(a_{2} \int_{0}^{\eta} \frac{(\eta-s)^{\alpha-\gamma-1}}{\Gamma(\alpha-\gamma)} f(s, x(s)) d s\right. \\
& \left.\quad+b_{2} \int_{0}^{T} \frac{(T-s)^{\alpha-\gamma-1}}{\Gamma(\alpha-\gamma)} f(s, x(s)) d s-c_{2}\right)+\frac{c_{1}}{a_{1}+b_{1}} .
\end{aligned}
$$

Note that problem (4) has solutions if and only if the operator $\mathcal{F} x$ has fixed points. We denote by $\mathcal{F} x=\mathcal{F}_{1} x+\mathcal{F}_{2} x$, where

$$
\left(\mathcal{F}_{1} x\right)(t)=\int_{0}^{t} \frac{(t-s)^{\alpha-1}}{\Gamma(\alpha)} f(s, x(s)) d s, \quad\left(\mathcal{F}_{2} x\right)(t)=-k_{1}^{x} t-k_{0}^{x} .
$$


Here the constants $k_{0}^{x}$ and $k_{1}^{x}$ are given by

$$
\begin{aligned}
k_{0}^{x}= & \frac{b_{1}}{a_{1}+b_{1}} \int_{0}^{T} \frac{(T-s)^{\alpha-1}}{\Gamma(\alpha)} f(s, x(s)) d s-\frac{c_{1}}{a_{1}+b_{1}}-\frac{b_{1} T \Gamma(2-\gamma)}{\left(a_{1}+b_{1}\right)\left(a_{2} \eta^{1-\gamma}+b_{2} T^{1-\gamma}\right)} \\
& \times\left(a_{2} \int_{0}^{\eta} \frac{(\eta-s)^{\alpha-\gamma-1}}{\Gamma(\alpha-\gamma)} f(s, x(s)) d s+b_{2} \int_{0}^{T} \frac{(T-s)^{\alpha-\gamma-1}}{\Gamma(\alpha-\gamma)} f(s, x(s)) d s-c_{2}\right), \\
k_{1}^{x}= & \frac{\Gamma(2-\gamma)\left(a_{2} \int_{0}^{\eta} \frac{(\eta-s)^{\alpha-\gamma-1}}{\Gamma(\alpha-\gamma)} f(s, x(s)) d s+b_{2} \int_{0}^{T} \frac{(T-s)^{\alpha-\gamma-1}}{\Gamma(\alpha-\gamma)} f(s, x(s)) d s-c_{2}\right)}{a_{2} \eta^{1-\gamma}+b_{2} T^{1-\gamma}} .
\end{aligned}
$$

Now, we are in a position to present our main results.

Theorem 3.1 Suppose that $f: J \times \mathbb{R} \rightarrow \mathbb{R}$ is continuous and satisfies

$$
|f(t, x)-f(t, y)| \leq m(t)|x-y|
$$

for $t \in J, x, y \in \mathbb{R}$, and $m \in L^{\infty}\left(J, \mathbb{R}^{+}\right)$. If

$$
(U+V)\left(1+\frac{\left|b_{1}\right|}{\left|a_{1}+b_{1}\right|}\right)<1
$$

then problem (4) has a unique solution, where

$$
\|m\|=\sup _{t \in J}|m(t)|, \quad U=\frac{T^{\alpha}\|m\|}{\Gamma(\alpha+1)}, \quad V=\frac{\|m\| \Gamma(2-\gamma)\left(T \eta^{\alpha-\gamma}\left|a_{2}\right|+T^{\alpha-\gamma+1}\left|b_{2}\right|\right)}{\Gamma(\alpha-\gamma+1)\left|a_{2} \eta^{1-\gamma}+b_{2} T^{1-\gamma}\right|} .
$$

Proof Denote $\mathcal{N}(x, y)=f(s, x(s))-f(s, y(s))$. For any $x, y \in \mathcal{C}$ and each $t \in J$, we have

$$
\begin{aligned}
& \left|\left(\mathcal{F}_{1} x\right)(t)-\left(\mathcal{F}_{1} y\right)(t)\right| \leq \int_{0}^{t} \frac{(t-s)^{\alpha-1}}{\Gamma(\alpha)}|\mathcal{N}(x, y)| d s \\
& \leq\|m\|\|x-y\| \int_{0}^{t} \frac{(t-s)^{\alpha-1}}{\Gamma(\alpha)} d s \\
& \leq U\|x-y\|, \\
& \left|\left(\mathcal{F}_{2} x\right)(t)-\left(\mathcal{F}_{2} y\right)(t)\right| \leq T\left|k_{1}^{x}-k_{1}^{y}\right|+\left|k_{0}^{x}-k_{0}^{y}\right|, \\
& T\left|k_{1}^{x}-k_{1}^{y}\right| \leq \frac{T \Gamma(2-\gamma)\left|a_{2}\right|}{\left|a_{2} \eta^{1-\gamma}+b_{2} T^{1-\gamma}\right|}\left|\int_{0}^{\eta} \frac{(\eta-s)^{\alpha-\gamma-1}}{\Gamma(\alpha-\gamma)} \mathcal{N}(x, y) d s\right| \\
& +\frac{T \Gamma(2-\gamma)\left|b_{2}\right|}{\left|a_{2} \eta^{1-\gamma}+b_{2} T^{1-\gamma}\right|}\left|\int_{0}^{T} \frac{(T-s)^{\alpha-\gamma-1}}{\Gamma(\alpha-\gamma)} \mathcal{N}(x, y) d s\right| \\
& \leq \frac{\|m\| T \Gamma(2-\gamma)\left|a_{2}\right|}{\left|a_{2} \eta^{1-\gamma}+b_{2} T^{1-\gamma}\right|}\|x-y\|+\frac{\|m\| T \Gamma(2-\gamma)\left|b_{2}\right|}{\left|a_{2} \eta^{1-\gamma}+b_{2} T^{1-\gamma}\right|}\|x-y\| \\
& =V\|x-y\|, \\
& \left|k_{0}^{x}-k_{0}^{y}\right| \leq\left|\frac{b_{1}}{a_{1}+b_{1}}\right|\left|\int_{0}^{T} \frac{(T-s)^{\alpha-1}}{\Gamma(\alpha)} \mathcal{N}(x, y) d s\right| \\
& +\frac{\left|b_{1} a_{2}\right| T \Gamma(2-\gamma)}{\left|a_{1}+b_{1}\right|\left|a_{2} \eta^{1-\gamma}+b_{2} T^{1-\gamma}\right|}\left|\int_{0}^{\eta} \frac{(\eta-s)^{\alpha-\gamma-1}}{\Gamma(\alpha-\gamma)} \mathcal{N}(x, y) d s\right|
\end{aligned}
$$




$$
\begin{aligned}
& +\frac{\left|b_{1} b_{2}\right| T \Gamma(2-\gamma)}{\left|a_{1}+b_{1}\right|\left|a_{2} \eta^{1-\gamma}+b_{2} T^{1-\gamma}\right|}\left|\int_{0}^{T} \frac{(T-s)^{\alpha-\gamma-1}}{\Gamma(\alpha-\gamma)} \mathcal{N}(x, y) d s\right| \\
\leq & \left(\frac{U\left|b_{1}\right|}{\left|a_{1}+b_{1}\right|}+\frac{V\left|b_{1}\right|}{\left|a_{1}+b_{1}\right|}\right)\|x-y\| .
\end{aligned}
$$

Therefore, we have

$$
\|(\mathcal{F} x)(t)-(\mathcal{F} y)(t)\| \leq(U+V)\left(1+\frac{\left|b_{1}\right|}{\left|a_{1}+b_{1}\right|}\right)\|x-y\| .
$$

This together with (8) implies that $\mathcal{F}$ is a contraction mapping. The contraction mapping principle yields that $\mathcal{F}$ has a unique fixed point, which is the unique solution of problem (4). This completes the proof.

Corollary 3.1 Suppose that $f: J \times \mathbb{R} \rightarrow \mathbb{R}$ is continuous and satisfies

$$
|f(t, x)-f(t, y)| \leq L|x-y|
$$

for $t \in J, x, y \in \mathbb{R}$, and $L>0$. Then problem (4) has a unique solution, provided

$$
\left(\frac{T^{\alpha} L}{\Gamma(\alpha+1)}+\frac{L \Gamma(2-\gamma)\left(T \eta^{\alpha-\gamma}\left|a_{2}\right|+T^{\alpha-\gamma+1}\left|b_{2}\right|\right)}{\Gamma(\alpha-\gamma+1)\left|a_{2} \eta^{1-\gamma}+b_{2} T^{1-\gamma}\right|}\right)\left(1+\frac{\left|b_{1}\right|}{\left|a_{1}+b_{1}\right|}\right)<1 .
$$

Theorem 3.2 Let $f: J \times \mathbb{R} \rightarrow \mathbb{R}$ be a continuous function. Assume that

$$
|f(t, x)| \leq m(t)+d|x|^{\rho}
$$

for each $t \in J, x \in \mathbb{R}, m \in L^{\infty}\left(J, \mathbb{R}^{+}\right), d \geq 0$ and $0 \leq \rho<1$. Then problem (4) has at least one solution.

Proof Let $B_{r}=\{x \in \mathcal{C}:\|x(t)\| \leq r$ and $t \in J\}, \mathcal{M}=\|m\|+d r^{\rho}$, where

$$
\begin{aligned}
r \geq & \max \left\{2 K,(2 L d)^{\frac{1}{1-\rho}}\right\}, \\
K= & \left(1+\frac{\left|b_{1}\right|}{\left|a_{1}+b_{1}\right|}\right)\left(\frac{T^{\alpha}\|m\|}{\Gamma(\alpha+1)}+\frac{T\|m\| \Gamma(2-\gamma)\left(\eta^{\alpha-\gamma}\left|a_{2}\right|+T^{\alpha-\gamma}\left|b_{2}\right|\right)}{\Gamma(\alpha-\gamma+1)\left|a_{2} \eta^{1-\gamma}+b_{2} T^{1-\gamma}\right|}\right) \\
& +\frac{T \Gamma(2-\gamma)\left|c_{2}\right|}{\left|a_{2} \eta^{1-\gamma}+b_{2} T^{1-\gamma}\right|}+\left|\frac{b_{1} c_{2} T \Gamma(2-\gamma)}{\left(a_{1}+b_{1}\right)\left(a_{2} \eta^{1-\gamma}+b_{2} T^{1-\gamma}\right)}-\frac{c_{1}}{a_{1}+b_{1}}\right|, \\
L= & \left(1+\frac{\left|b_{1}\right|}{\left|a_{1}+b_{1}\right|}\right)\left(\frac{T^{\alpha}}{\Gamma(\alpha+1)}+\frac{T \Gamma(2-\gamma)\left(\eta^{\alpha-\gamma}\left|a_{2}\right|+T^{\alpha-\gamma}\left|b_{2}\right|\right)}{\Gamma(\alpha-\gamma+1)\left|a_{2} \eta^{1-\gamma}+b_{2} T^{1-\gamma}\right|}\right) .
\end{aligned}
$$

Observe that $B_{r}$ is a closed, bounded convex subset of the Banach space $\mathcal{C}$.

Firstly, we prove that $\mathcal{F}: B_{r} \rightarrow B_{r}$. For any $x \in B_{r}$, we have

$$
\begin{aligned}
& \left|\left(\mathcal{F}_{1} x\right)(t)\right| \leq \int_{0}^{t} \frac{(t-s)^{\alpha-1}}{\Gamma(\alpha)}\left(m(s)+d|x(s)|^{\rho}\right) d s \leq \frac{T^{\alpha} \mathcal{M}}{\Gamma(\alpha+1)} \\
& T\left|k_{1}^{x}\right| \leq \frac{T \Gamma(2-\gamma)\left|c_{2}\right|}{\left|a_{2} \eta^{1-\gamma}+b_{2} T^{1-\gamma}\right|}+\frac{T \Gamma(2-\gamma)\left|a_{2} \int_{0}^{\eta}(\eta-s)^{\alpha-\gamma-1} f(s, x(s)) d s\right|}{\Gamma(\alpha-\gamma)\left|a_{2} \eta^{1-\gamma}+b_{2} T^{1-\gamma}\right|}
\end{aligned}
$$




$$
\begin{aligned}
& +\frac{T \Gamma(2-\gamma)\left|b_{2} \int_{0}^{T}(T-s)^{\alpha-\gamma-1} f(s, x(s)) d s\right|}{\Gamma(\alpha-\gamma)\left|a_{2} \eta^{1-\gamma}+b_{2} T^{1-\gamma}\right|} \\
\leq & \frac{T \Gamma(2-\gamma)\left|c_{2}\right|}{\left|a_{2} \eta^{1-\gamma}+b_{2} T^{1-\gamma}\right|}+\frac{T \mathcal{M} \Gamma(2-\gamma)\left(\eta^{\alpha-\gamma}\left|a_{2}\right|+T^{\alpha-\gamma}\left|b_{2}\right|\right)}{\Gamma(\alpha-\gamma+1)\left|a_{2} \eta^{1-\gamma}+b_{2} T^{1-\gamma}\right|}, \\
\left|k_{0}^{x}\right| \leq & \left|\frac{b_{1} c_{2} T \Gamma(2-\gamma)}{\left(a_{1}+b_{1}\right)\left(a_{2} \eta^{1-\gamma}+b_{2} T^{1-\gamma}\right)}-\frac{c_{1}}{a_{1}+b_{1}}\right| \\
& +\left|\frac{b_{1}}{a_{1}+b_{1}} \int_{0}^{T} \frac{(T-s)^{\alpha-1}}{\Gamma(\alpha)} f(s, x(s)) d s\right|+\frac{T \Gamma(2-\gamma)\left|b_{1}\right|}{\left|\left(a_{1}+b_{1}\right)\left(a_{2} \eta^{1-\gamma}+b_{2} T^{1-\gamma}\right)\right|} \\
& \times\left(\left|a_{2} \int_{0}^{\eta} \frac{(\eta-s)^{\alpha-\gamma-1}}{\Gamma(\alpha-\gamma)} f(s, x(s)) d s\right|+\left|b_{2} \int_{0}^{T} \frac{(T-s)^{\alpha-\gamma-1}}{\Gamma(\alpha-\gamma)} f(s, x(s)) d s\right|\right) \\
\leq & \left|\frac{b_{1} c_{2} T \Gamma(2-\gamma)}{\left(a_{1}+b_{1}\right)\left(a_{2} \eta^{1-\gamma}+b_{2} T^{1-\gamma}\right)}-\frac{c_{1}}{a_{1}+b_{1}}\right|+\frac{T^{\alpha} \mathcal{M}\left|b_{1}\right|}{\Gamma(\alpha+1)\left|a_{1}+b_{1}\right|} \\
& +\frac{T \mathcal{M} \Gamma(2-\gamma)\left|b_{1}\right|\left(\eta^{\alpha-\gamma}\left|a_{2}\right|+T^{\alpha-\gamma}\left|b_{2}\right|\right)}{\Gamma(\alpha-\gamma+1)\left|\left(a_{1}+b_{1}\right)\left(a_{2} \eta^{1-\gamma}+b_{2} T^{1-\gamma}\right)\right|} .
\end{aligned}
$$

Hence, we have

$$
\begin{aligned}
\|\mathcal{F} x\| \leq & \left(1+\frac{\left|b_{1}\right|}{\left|a_{1}+b_{1}\right|}\right)\left(\frac{T^{\alpha}\|m\|}{\Gamma(\alpha+1)}+\frac{T\|m\| \Gamma(2-\gamma)\left(\eta^{\alpha-\gamma}\left|a_{2}\right|+T^{\alpha-\gamma}\left|b_{2}\right|\right)}{\Gamma(\alpha-\gamma+1)\left|a_{2} \eta^{1-\gamma}+b_{2} T^{1-\gamma}\right|}\right) \\
& +\frac{T \Gamma(2-\gamma)\left|c_{2}\right|}{\left|a_{2} \eta^{1-\gamma}+b_{2} T^{1-\gamma}\right|}+\left|\frac{b_{1} c_{2} T \Gamma(2-\gamma)}{\left(a_{1}+b_{1}\right)\left(a_{2} \eta^{1-\gamma}+b_{2} T^{1-\gamma}\right)}-\frac{c_{1}}{a_{1}+b_{1}}\right| \\
& +d r^{\rho}\left(1+\frac{\left|b_{1}\right|}{\left|a_{1}+b_{1}\right|}\right)\left(\frac{T^{\alpha}}{\Gamma(\alpha+1)}+\frac{T \Gamma(2-\gamma)\left(\eta^{\alpha-\gamma}\left|a_{2}\right|+T^{\alpha-\gamma}\left|b_{2}\right|\right)}{\Gamma(\alpha-\gamma+1)\left|a_{2} \eta^{1-\gamma}+b_{2} T^{1-\gamma}\right|}\right) \\
\leq & K+d r^{\rho} L \leq \frac{r}{2}+\frac{r}{2}=r .
\end{aligned}
$$

This implies that $\mathcal{F}: B_{r} \rightarrow B_{r}$.

Secondly, we prove that $\mathcal{F}$ maps bounded sets into equicontinuous sets. Let $B$ be any bounded set of $\mathcal{C}$. Notice that $f$ is continuous on $J$, therefore, without loss of generality, we can assume that there is an $N$ such that

$$
|f(t, x(t))| \leq N
$$

for any $t \in J$ and $x \in B$. Now, we let $0 \leq t_{1} \leq t_{2} \leq T$. Then for each $x \in B$, we have

$$
\begin{aligned}
& \left|\left(\mathcal{F}_{1} x\right)\left(t_{2}\right)-\left(\mathcal{F}_{1} x\right)\left(t_{1}\right)\right| \\
& \quad \leq\left|\int_{0}^{t_{1}} \frac{\left(t_{2}-s\right)^{\alpha-1}-\left(t_{1}-s\right)^{\alpha-1}}{\Gamma(\alpha)} f(s, x(s)) d s\right|+\left|\int_{t_{1}}^{t_{2}} \frac{\left(t_{2}-s\right)^{\alpha-1}}{\Gamma(\alpha)} f(s, x(s)) d s\right| \\
& \quad \leq \frac{N\left(t_{2}-t_{1}\right)^{\alpha}}{\Gamma(\alpha+1)}+\frac{N\left(t_{1}^{\alpha}+\left(t_{2}-t_{1}\right)^{\alpha}-t_{2}^{\alpha}\right)}{\Gamma(\alpha+1)} \leq \frac{2 N\left(t_{2}-t_{1}\right)^{\alpha}}{\Gamma(\alpha+1)}
\end{aligned}
$$

and

$$
\begin{aligned}
& \left|\left(\mathcal{F}_{2} x\right)\left(t_{2}\right)-\left(\mathcal{F}_{2} x\right)\left(t_{1}\right)\right| \\
& \quad \leq\left|k_{1}^{x}\right|\left(t_{2}-t_{1}\right) \\
& \quad \leq \frac{\Gamma(2-\gamma)\left(N \eta^{\alpha-\gamma}\left|a_{2}\right|+N T^{\alpha-\gamma}\left|b_{2}\right|+\Gamma(\alpha-\gamma+1)\left|c_{2}\right|\right)\left(t_{2}-t_{1}\right)}{\Gamma(\alpha-\gamma+1)\left|a_{2} \eta^{1-\gamma}+b_{2} T^{1-\gamma}\right|} .
\end{aligned}
$$


Hence, we have

$$
\left\|(\mathcal{F} x)\left(t_{2}\right)-(\mathcal{F} x)\left(t_{1}\right)\right\| \rightarrow 0 \quad \text { as } t_{2} \rightarrow t_{1},
$$

and the limit is independent of $x \in B$. Therefore, the operator $\mathcal{F}: B_{r} \rightarrow B_{r}$ is equicontinuous and uniformly bounded. The Arzela-Ascoli theorem implies that $\mathcal{F}\left(B_{r}\right)$ is relatively compact in $\mathcal{C}$. By Theorem 2.1, we know that problem (4) has at least one solution. The proof is completed.

Corollary 3.2 Assume that $|f(t, x)| \leq v(t)$ for $t \in J, x \in \mathbb{R}$ with $v \in C\left(J\right.$, $\left.\mathbb{R}^{+}\right)$. Then problem (4) has at least one solution.

Theorem 3.3 Let $f: J \times \mathbb{R} \rightarrow \mathbb{R}$ be a continuous function. Assume that

(1) there exists a function $m \in L^{\infty}\left(J, \mathbb{R}^{+}\right)$and a non-decreasing function $\varphi:[0, \infty) \rightarrow[0, \infty)$ such that

$$
|f(t, x)| \leq m(t) \varphi(\|x\|)
$$

where $t \in J, x \in \mathbb{R}$;

(2) there exists a constant $K>0$ such that

$$
\frac{K}{R+\varphi(K) Q}>1,
$$

where

$$
\begin{aligned}
R= & \frac{T \Gamma(2-\gamma)\left|c_{2}\right|}{\left|a_{2} \eta^{1-\gamma}+b_{2} T^{1-\gamma}\right|}+\left|\frac{c_{1}}{a_{1}+b_{1}}-\frac{b_{1} c_{2} T \Gamma(2-\gamma)}{\left(a_{1}+b_{1}\right)\left(a_{2} \eta^{1-\gamma}+b_{2} T^{1-\gamma}\right)}\right|, \\
Q= & \frac{T^{\alpha}\|m\|}{\Gamma(\alpha+1)}+\frac{T \Gamma(2-\gamma)\|m\|\left(\left|a_{2}\right| \eta^{1-\gamma}+\left|b_{2}\right| T^{1-\gamma}\right)}{\Gamma(\alpha-\gamma+1)\left|a_{2} \eta^{1-\gamma}+b_{2} T^{1-\gamma}\right|} \\
& +\frac{\|m\|\left|b_{1}\right|}{\left|a_{1}+b_{1}\right|}\left(\frac{T \Gamma(2-\gamma)\left(\left|a_{2}\right| \eta^{1-\gamma}+\left|b_{2}\right| T^{1-\gamma}\right)}{\Gamma(\alpha-\gamma+1)\left|a_{2} \eta^{1-\gamma}+b_{2} T^{1-\gamma}\right|}+\frac{T^{\alpha}}{\Gamma(\alpha+1)}\right) .
\end{aligned}
$$

Then problem (4) has at least one solution.

Proof Firstly, we prove that $\mathcal{F}$ maps bounded sets into bounded sets in $\mathcal{C}$. Let $B$ be a bounded subset of $\mathcal{C}$ and $\|x\| \leq r$ for any $x \in B$. As in the proof of Theorem 3.2, we have

$$
\begin{aligned}
& \left|\left(\mathcal{F}_{1} x\right)(t)\right| \leq\left|\int_{0}^{t} \frac{(t-s)^{\alpha-1}}{\Gamma(\alpha)} f(s, x(s))\right| d s \leq \frac{T^{\alpha}\|m\| \varphi(r)}{\Gamma(\alpha+1)}, \\
& \left|\left(\mathcal{F}_{2} x\right)(t)\right| \leq T\left|k_{1}^{x}\right|+\left|k_{0}^{x}\right|, \\
& T\left|k_{1}^{x}\right| \leq \frac{T \Gamma(2-\gamma)\|m\| \varphi(r)\left(\left|a_{2}\right| \eta^{1-\gamma}+\left|b_{2}\right| T^{1-\gamma}\right)}{\Gamma(\alpha-\gamma+1)\left|a_{2} \eta^{1-\gamma}+b_{2} T^{1-\gamma}\right|}+\frac{T \Gamma(2-\gamma)\left|c_{2}\right|}{\left|a_{2} \eta^{1-\gamma}+b_{2} T^{1-\gamma}\right|}, \\
& \left|k_{0}^{x}\right| \leq \frac{\|m\| \varphi(r)\left|b_{1}\right|}{\left|a_{1}+b_{1}\right|}\left(\frac{T \Gamma(2-\gamma)\left(\left|a_{2}\right| \eta^{1-\gamma}+\left|b_{2}\right| T^{1-\gamma}\right)}{\Gamma(\alpha-\gamma+1)\left|a_{2} \eta^{1-\gamma}+b_{2} T^{1-\gamma}\right|}+\frac{T^{\alpha}}{\Gamma(\alpha+1)}\right) \\
& \quad+\left|\frac{c_{1}}{a_{1}+b_{1}}-\frac{b_{1} c_{2} T \Gamma(2-\gamma)}{\left(a_{1}+b_{1}\right)\left(a_{2} \eta^{1-\gamma}+b_{2} T^{1-\gamma}\right)}\right| .
\end{aligned}
$$


Hence,

$$
\|\mathcal{F} x\| \leq R+\varphi(r) Q
$$

Secondly, we claim that $\mathcal{F}$ is equicontinuous. The proof of this claim is the same as the one in the proof of Theorem 3.2.

Finally, we let $x=\lambda \mathcal{F} x$ for some $\lambda \in(0,1)$. Then for each $t \in J$, we have

$$
|x|=|\lambda \mathcal{F} x| \leq R+\varphi(\|x\|) Q
$$

This implies that

$$
\frac{\|x\|}{R+\varphi(\|x\|) Q} \leq 1
$$

According to the assumptions, we know that there exists $K$ such that $K \neq\|x\|$. Let

$$
O=\{y \in \mathcal{C}:\|y\|<K\} .
$$

The operator $\mathcal{F}: \bar{O} \rightarrow \mathcal{C}$ is continuous and completely continuous. Combining the choice of $O$ and Theorem 2.2, we can deduce that $\mathcal{F}$ has a fixed point in $\bar{O}$, which is a solution of problem (4).

\section{Existence results for problem (5)}

Lemma 4.1 For any $y \in C([0,1], \mathbb{R})$, the unique solution of the three-point boundary value problem

$$
\left\{\begin{array}{l}
{ }^{c} D^{\alpha} x(t)=y(t), \quad t \in[0,1], 1<\alpha \leq 2, \\
x(0)=0, \quad a I^{\gamma} x(\eta)+b x(1)=c, \quad 0<\eta<1,
\end{array}\right.
$$

is given by

$$
\begin{aligned}
x(t)= & \int_{0}^{t} \frac{(t-s)^{\alpha-1}}{\Gamma(\alpha)} y(s) d s+\frac{t\left(c-b \int_{0}^{1} \frac{(1-s)^{\alpha-1}}{\Gamma(\alpha)} y(s) d s\right)}{\frac{a \eta^{1+\gamma}}{\Gamma(\gamma+2)}+b} \\
& -\frac{\operatorname{ta} \int_{0}^{\eta} \frac{(\eta-s)^{\alpha+\gamma-1}}{\Gamma(\alpha+\gamma)} y(s) d s}{\frac{a \eta^{1+\gamma}}{\Gamma(\gamma+2)}+b} .
\end{aligned}
$$

Proof For $1<\alpha \leq 2$ and some constants $c_{0}, c_{1} \in \mathbb{R}$, the general solution of the equation ${ }^{c} D^{\alpha} x(t)=y(t)$ can be written as

$$
x(t)=I^{\alpha} y(t)+c_{0}+c_{1} t .
$$

From $x(0)=0$, it follows that $c_{0}=0$. Using the integral boundary conditions of (9), we obtain

$$
\left(\frac{a \eta^{1+\gamma}}{\Gamma(\gamma+2)}+b\right) c_{1}+a I^{\alpha+\gamma} y(\eta)+b \int_{0}^{1} \frac{(1-s)^{\alpha-1}}{\Gamma(\alpha)} y(s) d s=c .
$$


Therefore, we have

$$
c_{1}=\frac{c-b \int_{0}^{1} \frac{(1-s)^{\alpha-1}}{\Gamma(\alpha)} y(s) d s-a \int_{0}^{\eta} \frac{(\eta-s)^{\alpha+\gamma-1}}{\Gamma(\alpha+\gamma)} y(s) d s}{\frac{a \eta^{1+\gamma}}{\Gamma(\gamma+2)}+b} .
$$

Substituting the values of $c_{0}, c_{1}$, we obtain the result. This completes the proof.

Define the space $X=\left\{x: x\right.$ and $\left.{ }^{c} D^{\beta} x \in C([0,1], \mathbb{R}), 0<\beta<1\right\}$ endowed with the norm $\|x\|_{*}=\max _{t \in[0,1]}|x(t)|+\max _{t \in[0,1]}\left|{ }^{c} D^{\beta} x(t)\right|$. Obviously, $\left(X,\|\cdot\|_{*}\right)$ is a Banach space. In order to obtain the existence results of problem (5), by Lemma 4.1, we define an operator $\mathcal{S}: X \rightarrow$ $X$ as follows

$$
\begin{aligned}
(\mathcal{S} x)(t)= & \int_{0}^{t} \frac{(t-s)^{\alpha-1}}{\Gamma(\alpha)}(N x)(s) d s+\frac{t\left(c-b \int_{0}^{1} \frac{(1-s)^{\alpha-1}}{\Gamma(\alpha)}(N x)(s) d s\right)}{\frac{a \eta^{1+\gamma}}{\Gamma(\gamma+2)}+b} \\
& -\frac{t a \int_{0}^{\eta} \frac{(\eta-s)^{\alpha+\gamma-1}}{\Gamma(\alpha+\gamma)}(N x)(s) d s}{\frac{a \eta^{1+\gamma}}{\Gamma(\gamma+2)}+b},
\end{aligned}
$$

where

$$
(N x)(t)=f\left(t, x(t),{ }^{c} D^{\beta} x(t)\right) .
$$

Since $f$ is continuous, it is easy to see that

$$
\left({ }^{c} D^{\beta} \mathcal{S} x\right)(t)=\left(I^{\alpha-\beta} N x\right)(t)-\frac{k t^{1-\beta}}{\Gamma(2-\beta)},
$$

here $k$ is a constant given by

$$
k=\frac{b \int_{0}^{1} \frac{(1-s)^{\alpha-1}}{\Gamma(\alpha)}(N x)(s) d s+a \int_{0}^{\eta} \frac{(\eta-s)^{\alpha+\gamma-1}}{\Gamma(\alpha+\gamma)}(N x)(s) d s-c}{\frac{a \eta^{1+\gamma}}{\Gamma(\gamma+2)}+b} .
$$

Theorem 4.1 Let $f:[0,1] \times \mathbb{R} \times \mathbb{R} \rightarrow \mathbb{R}$ be a continuous function satisfying that

$$
\left|f\left(t, x_{1}, y_{1}\right)-f\left(t, x_{2}, y_{2}\right)\right| \leq m(t)\left(\left|x_{1}-x_{2}\right|+\left|y_{1}-y_{2}\right|\right)
$$

for $t \in[0,1], x_{i}, y_{i} \in \mathbb{R}, i=1,2$ and $m(t) \in L^{\frac{1}{\tau}}\left([0,1], \mathbb{R}^{+}\right), \tau \in(0, \alpha-1)$. Then problem (5) has a unique solution provided that $\Delta+\Lambda<1$, where $\Delta, \Lambda$ are given by

$$
\begin{aligned}
\Delta= & \frac{\|m\|\left(\frac{1-\tau}{\alpha-\tau}\right)^{1-\tau}}{\Gamma(\alpha)}\left(1+\frac{|b|}{\left|\frac{a \eta^{1+\gamma}}{\Gamma(\gamma+2)}+b\right|}\right)+\frac{\|m\||a| \eta^{\alpha+\gamma-\tau}\left(\frac{1-\tau}{\alpha+\gamma-\tau}\right)^{1-\tau}}{\Gamma(\alpha+\gamma)\left|\frac{a \eta^{1+\gamma}}{\Gamma(\gamma+2)}+b\right|}, \\
\Lambda= & \frac{\|m\|}{\Gamma(2-\beta)}\left(\frac{|b|}{\Gamma(\alpha)}\left(\frac{1-\tau}{\alpha-\tau}\right)^{1-\tau}+\frac{|a| \eta^{\alpha+\gamma-\tau}}{\Gamma(\alpha+\gamma)}\left(\frac{1-\tau}{\alpha+\gamma-\tau}\right)^{1-\tau}\right) \\
& +\frac{\|m\|\left(\frac{1-\tau}{\alpha-\beta-\tau}\right)^{1-\tau}}{\Gamma(\alpha-\beta)} .
\end{aligned}
$$


Proof Let $x, y \in X$ and $\|m\|=\left(\int_{0}^{1}|m(s)|^{\frac{1}{\tau}} d s\right)^{\tau}$. Then for each $t \in[0,1]$, we have

$$
\begin{aligned}
|(\mathcal{S} x)(t)-(\mathcal{S} y)(t)| \leq & \int_{0}^{t} \frac{(t-s)^{\alpha-1}}{\Gamma(\alpha)}|(N x)(s)-N(y)(s)| d s \\
& +\frac{|b| \int_{0}^{1}(1-s)^{\alpha-1}|(N x)(s)-N(y)(s)| d s}{\Gamma(\alpha)\left|\frac{a \eta^{1+\gamma}}{\Gamma(\gamma+2)}+b\right|} \\
& +\frac{|a| \int_{0}^{\eta}(\eta-s)^{\alpha+\gamma-1}|(N x)(s)-N(y)(s)| d s}{\Gamma(\alpha+\gamma)\left|\frac{a \eta^{1+\gamma}}{\Gamma(\gamma+2)}+b\right|} \\
\leq & \int_{0}^{t} \frac{(t-s)^{\alpha-1}}{\Gamma(\alpha)} m(s)\|x-y\|_{*} d s+\frac{|b| \int_{0}^{1}(1-s)^{\alpha-1} m(s)\|x-y\|_{*} d s}{\Gamma(\alpha)\left|\frac{a \eta^{1+\gamma}}{\Gamma(\gamma+2)}+b\right|} \\
& +\frac{|a| \int_{0}^{\eta}(\eta-s)^{\alpha+\gamma-1} m(s)\|x-y\|_{*} d s}{\Gamma(\alpha+\gamma)\left|\frac{a \eta^{1+\gamma}}{\Gamma(\gamma+2)}+b\right|} .
\end{aligned}
$$

By the Hölder inequality, we have

$$
\begin{aligned}
& |(\mathcal{S} x)(t)-(\mathcal{S} y)(t)| \\
& \quad \leq\left\{\frac{\|m\|\left(\frac{1-\tau}{\alpha-\tau}\right)^{1-\tau}}{\Gamma(\alpha)}\left(1+\frac{|b|}{\left|\frac{a \eta^{1+\gamma}}{\Gamma(\gamma+2)}+b\right|}\right)+\frac{\|m\||a| \eta^{\alpha+\gamma-\tau}\left(\frac{1-\tau}{\alpha+\gamma-\tau}\right)^{1-\tau}}{\Gamma(\alpha+\gamma)\left|\frac{a \eta^{1+\gamma}}{\Gamma(\gamma+2)}+b\right|}\right\}\|x-y\|_{*} \\
& \quad=\Delta\|x-y\|_{*} .
\end{aligned}
$$

Similarly, we have

$$
\begin{aligned}
& \left|\left({ }^{c} D^{\beta} \mathcal{S} x\right)(t)-\left({ }^{c} D^{\beta} \mathcal{S} y\right)(t)\right| \\
& \leq \frac{\|m\|}{\Gamma(2-\beta)}\left(\frac{|b|}{\Gamma(\alpha)}\left(\frac{1-\tau}{\alpha-\tau}\right)^{1-\tau}+\frac{|a| \eta^{\alpha+\gamma-\tau}}{\Gamma(\alpha+\gamma)}\left(\frac{1-\tau}{\alpha+\gamma-\tau}\right)^{1-\tau}\right)\|x-y\|_{*} \\
& \quad+\frac{\|m\|\left(\frac{1-\tau}{\alpha-\beta-\tau}\right)^{1-\tau}\|x-y\|_{*}}{\Gamma(\alpha-\beta)} \\
& =\Lambda\|x-y\|_{*} .
\end{aligned}
$$

From the inequalities above, we can deduce that

$$
\|(\mathcal{S} x)(t)-(\mathcal{S} y)(t)\|_{*} \leq(\Delta+\Lambda)\|x-y\|_{*}
$$

By the contraction principle, we know that problem (5) has a unique solution.

\section{Theorem 4.2 Assume that}

(1) there exist two non-decreasing functions $\rho_{1}, \rho_{2}:[0, \infty) \rightarrow[0, \infty)$ and a function $m \in L^{\frac{1}{\tau}}\left([0,1], \mathbb{R}^{+}\right)$with $\tau \in(0, \alpha-1)$ such that

$$
|f(t, x, y)| \leq m(t)\left(\rho_{1}(|x|)+\rho_{2}(|y|)\right)
$$

for $t \in[0,1]$ and $x, y \in \mathbb{R}$; 
(2) there exists a constant $Z>0$ such that

$$
\frac{Z}{\frac{|c|(1+\Gamma(2-\beta))}{\Gamma(2-\beta)\left|\frac{a \eta^{1+\gamma}}{\Gamma(\gamma+2)}+b\right|}+\|m\| W\left(\rho_{1}(Z)+\rho_{2}(Z)\right)}>1,
$$

where $\|m\|=\left(\int_{0}^{1}|m(s)|^{\frac{1}{\tau}} d s\right)^{\tau}$ and

$$
\begin{aligned}
W= & \frac{1}{\Gamma(\alpha)}\left(1+\frac{|b|}{\left|\frac{a \eta^{1+\gamma}}{\Gamma(\gamma+2)}+b\right|}+\frac{|b|}{\Gamma(2-\beta)\left|\frac{a \eta^{1+\gamma}}{\Gamma(\gamma+2)}+b\right|}\right)\left(\frac{1-\tau}{\alpha-\tau}\right)^{1-\tau} \\
& +\frac{|a| \eta^{\alpha+\gamma-\tau}}{\Gamma(\alpha+\gamma)\left|\frac{a \eta^{1+\gamma}}{\Gamma(\gamma+2)}+b\right|}\left(1+\frac{1}{\Gamma(2-\beta)}\right)\left(\frac{1-\tau}{\alpha+\gamma-\tau}\right)^{1-\tau} \\
& +\frac{1}{\Gamma(\alpha-\beta)}\left(\frac{1-\tau}{\alpha-\beta-\tau}\right)^{1-\tau} .
\end{aligned}
$$

Then problem (5) has at least one solution on $[0,1]$.

Proof The proof consists of the following steps.

Firstly, we show that the operator $\mathcal{S}: X \rightarrow X$ maps bounded sets into bounded sets. Let $B_{r}=\left\{x \in X:\|x\|_{*} \leq r\right\}$ be a bounded set in $X$. Then for each $x \in B_{r}$, we have

$$
\begin{aligned}
|\mathcal{S} x| \leq & \int_{0}^{t} \frac{(t-s)^{\alpha-1}}{\Gamma(\alpha)}|(N x)(s)| d s+\frac{\left(|c|+|b| \int_{0}^{1} \frac{(1-s)^{\alpha-1}}{\Gamma(\alpha)}|(N x)(s)| d s\right)}{\left|\frac{a \eta^{1+\gamma}}{\Gamma(\gamma+2)}+b\right|} \\
& +\frac{|a| \int_{0}^{\eta} \frac{(\eta-s)^{\alpha+\gamma-1}}{\Gamma(\alpha+\gamma)}|(N x)(s)| d s}{\left|\frac{a \eta^{1+\gamma}}{\Gamma(\gamma+2)}+b\right|} \\
\leq & \frac{\rho_{1}(r)+\rho_{2}(r)}{\Gamma(\alpha)} \int_{0}^{t}(t-s)^{\alpha-1} m(s) d s+\frac{|c|}{\left|\frac{a \eta^{1+\gamma}}{\Gamma(\gamma+2)}+b\right|} \\
& +\frac{\left(\rho_{1}(r)+\rho_{2}(r)\right)\left(|a| \int_{0}^{\eta} \frac{(\eta-s)^{\alpha+\gamma-1}}{\Gamma(\alpha+\gamma)} m(s) d s+|b| \int_{0}^{1} \frac{(1-s)^{\alpha-1}}{\Gamma(\alpha)} m(s) d s\right)}{\left|\frac{a \eta^{1+\gamma}}{\Gamma(\gamma+2)}+b\right|} .
\end{aligned}
$$

By using the Hölder inequality, we have

$$
\begin{aligned}
|\mathcal{S} x| \leq & \frac{\|m\|\left(\rho_{1}(r)+\rho_{2}(r)\right)\left(\frac{1-\tau}{\alpha-\tau}\right)^{1-\tau}}{\Gamma(\alpha)}\left(1+\frac{|b|}{\left|\frac{a \eta^{1+\gamma}}{\Gamma(\gamma+2)}+b\right|}\right)+\frac{|c|}{\left|\frac{a \eta^{1+\gamma}}{\Gamma(\gamma+2)}+b\right|} \\
& +\frac{|a|\|m\| \eta^{\alpha+\gamma-\tau}\left(\rho_{1}(r)+\rho_{2}(r)\right)}{\Gamma(\alpha+\gamma)\left|\frac{a \eta^{1+\gamma}}{\Gamma(\gamma+2)}+b\right|}\left(\frac{1-\tau}{\alpha+\gamma-\tau}\right)^{1-\tau} \cdot
\end{aligned}
$$

Similarly, we can obtain that

$$
\begin{aligned}
& \left|\left({ }^{c} D^{\beta} \mathcal{S} x\right)(t)\right| \\
& \quad \leq\left|\left(I^{\alpha-\beta} N x\right)(t)\right|+\frac{|k|}{\Gamma(2-\beta)}
\end{aligned}
$$




$$
\begin{aligned}
\leq & \frac{\|m\|\left(\rho_{1}(r)+\rho_{2}(r)\right)}{\Gamma(\alpha-\beta)}\left(\frac{1-\tau}{\alpha-\beta-\tau}\right)^{1-\tau}+\frac{|b|\|m\|\left(\rho_{1}(r)+\rho_{2}(r)\right)}{\Gamma(2-\beta) \Gamma(\alpha)\left|\frac{a \eta^{1+\gamma}}{\Gamma(\gamma+2)}+b\right|}\left(\frac{1-\tau}{\alpha-\tau}\right)^{1-\tau} \\
& +\frac{|a|\|m\| \eta^{\alpha+\gamma-\tau}\left(\rho_{1}(r)+\rho_{2}(r)\right)}{\Gamma(2-\beta) \Gamma(\alpha+\gamma)\left|\frac{a \eta^{1+\gamma}}{\Gamma(\gamma+2)}+b\right|}\left(\frac{1-\tau}{\alpha+\gamma-\tau}\right)^{1-\tau}+\frac{|c|}{\Gamma(2-\beta)\left|\frac{a \eta^{1+\gamma}}{\Gamma(\gamma+2)}+b\right|} .
\end{aligned}
$$

Therefore, we have

$$
\begin{aligned}
& \|(\mathcal{S} x)(t)\|_{*} \\
& \leq \frac{\|m\|\left(\rho_{1}(r)+\rho_{2}(r)\right)\left(\frac{1-\tau}{\alpha-\tau}\right)^{1-\tau}}{\Gamma(\alpha)}\left(1+\frac{|b|}{\left|\frac{a \eta^{1+\gamma}}{\Gamma(\gamma+2)}+b\right|}+\frac{|b|}{\Gamma(2-\beta)\left|\frac{a \eta^{1+\gamma}}{\Gamma(\gamma+2)}+b\right|}\right) \\
& \quad+\frac{\|m\|\left(\rho_{1}(r)+\rho_{2}(r)\right)}{\Gamma(\alpha-\beta)}\left(\frac{1-\tau}{\alpha-\beta-\tau}\right)^{1-\tau}+\frac{|c|}{\left|\frac{a \eta^{1+\gamma}}{\Gamma(\gamma+2)}+b\right|}\left(1+\frac{1}{\Gamma(2-\beta)}\right) \\
& \quad+\frac{|a|\|m\| \eta^{\alpha+\gamma-\tau}\left(\rho_{1}(r)+\rho_{2}(r)\right)}{\Gamma(\alpha+\gamma)\left|\frac{a \eta^{1+\gamma}}{\Gamma(\gamma+2)}+b\right|}\left(1+\frac{1}{\Gamma(2-\beta)}\right)\left(\frac{1-\tau}{\alpha+\gamma-\tau}\right)^{1-\tau} .
\end{aligned}
$$

That is to say, we have

$$
\|(\mathcal{S} x)(t)\|_{*} \leq \frac{|c|(1+\Gamma(2-\beta))}{\Gamma(2-\beta)\left|\frac{a \eta^{1+\gamma}}{\Gamma(\gamma+2)}+b\right|}+\|m\| W\left(\rho_{1}(r)+\rho_{2}(r)\right) .
$$

Secondly, by a discussion similar to that of Theorem 3.2, we can get

$$
\begin{aligned}
& \left|(\mathcal{S} x)\left(t_{2}\right)-(\mathcal{S} x)\left(t_{1}\right)\right| \rightarrow 0, \\
& \left|\left({ }^{c} D^{\beta} \mathcal{S} x\right)\left(t_{2}\right)-\left({ }^{c} D^{\beta} \mathcal{S} x\right)(t)\left(t_{1}\right)\right| \rightarrow 0
\end{aligned}
$$

as $t_{2} \rightarrow t_{1}$. This implies that

$$
\left\|(\mathcal{S} x)\left(t_{2}\right)-(\mathcal{S} x)\left(t_{1}\right)\right\|_{*} \rightarrow 0 \quad \text { as } t_{2} \rightarrow t_{1}
$$

Finally, we let $x=\lambda \mathcal{S} x$ for $\lambda \in(0,1)$. Then for each $t \in[0,1]$, we have

$$
\|x\|_{*}=\|\lambda \mathcal{S} x\|_{*} \leq \frac{|c|(1+\Gamma(2-\beta))}{\Gamma(2-\beta)\left|\frac{a \eta^{1+\gamma}}{\Gamma(\gamma+2)}+b\right|}+\|m\| W\left(\rho_{1}\left(\|x\|_{*}\right)+\rho_{2}\left(\|x\|_{*}\right)\right) .
$$

That is to say,

$$
\frac{\|x\|_{*}}{\frac{|c|(1+\Gamma(2-\beta))}{\Gamma(2-\beta)\left|\frac{a^{1+\gamma}}{\Gamma(\gamma+2)}+b\right|}+\|m\| W\left(\rho_{1}\left(\|x\|_{*}\right)+\rho_{2}\left(\|x\|_{*}\right)\right)} \leq 1 .
$$

By the assumptions and a discussion similar to the one in the proof of Theorem 3.3, we can deduce that $\mathcal{S}$ has a fixed point in $X$. So the proof of this theorem is completed.

\section{Examples}

In this section, we give two examples to illustrate the main results. 
Example 1 Consider the boundary value problem

$$
\left\{\begin{array}{l}
{ }^{c} D^{\frac{5}{3}} x(t)=\left(5 t^{2}-3 t\right) e^{-x^{2}(t)}+\frac{1}{3 \pi}|x(t)|^{\frac{1}{4}}, \quad t \in[0,1], \\
3 x(0)+\frac{1}{2} x(1)=2, \quad{ }^{c} D^{\frac{1}{2}} x\left(\frac{1}{4}\right)+\frac{1}{3}\left({ }^{c} D^{\frac{1}{2}} x(1)\right)=-\frac{1}{3} .
\end{array}\right.
$$

Here $\alpha=\frac{5}{3}, \gamma=\frac{1}{2}, a_{1}=3, b_{1}=\frac{1}{2}, c_{1}=2, a_{2}=1, b_{2}=\frac{1}{3}, c_{2}=-\frac{1}{3}, T=1$ and

$$
f(t, x)=\left(5 t^{2}-3 t\right) e^{-x^{2}(t)}+\frac{1}{3 \pi}|x(t)|^{\frac{1}{4}} .
$$

Since

$$
|f(t, x)| \leq\left|5 t^{2}-3 t\right|+\frac{1}{3 \pi}|x|^{\frac{1}{4}}
$$

let $d=\frac{1}{3 \pi}, \rho=\frac{1}{4}$ and $m(t)=\left|5 t^{2}-3 t\right|$. Thus, by Theorem 3.2, problem (11) has at least one solution on $[0,1]$.

Example 2 Consider the following fractional differential equation

$$
\left\{\begin{array}{l}
{ }^{c} D^{\frac{3}{2}} x(t)=\frac{e^{-x^{2}(t)}}{(5+t)^{2}} \frac{|x(t)|}{1+|x(t)|}+\frac{\left.\right|^{c} D^{\frac{3}{4}} x(t) \mid}{\left(4+\sin ^{2} x(t)\right)^{2}}, \quad t \in[0,1], \\
x(0)=0, \quad \sqrt{2}\left[I^{\frac{5}{2}} x\right]\left(\frac{1}{3}\right)+x(1)=2 .
\end{array}\right.
$$

In this case $\alpha=\frac{3}{2}, \beta=\frac{3}{4}, \gamma=\frac{5}{2}, a=\sqrt{2}, b=1, c=2, \eta=\frac{1}{3}$ and

$$
f\left(t, x,{ }^{c} D^{\frac{3}{4}} x\right)=\frac{e^{-x^{2}}}{(5+t)^{2}} \frac{|x|}{1+|x|}+\frac{{ }^{c} D^{\frac{3}{4}} x}{\left(4+\sin ^{2} x\right)^{2}} .
$$

Since

$$
\left|f\left(t, x,{ }^{c} D^{\frac{3}{4}} x\right)-f\left(t, y,{ }^{c} D^{\frac{3}{4}} y\right)\right| \leq \frac{1}{16}\left(|x-y|+\left|{ }^{c} D^{\frac{3}{4}} x-{ }^{c} D^{\frac{3}{4}} y\right|\right),
$$

let $\tau=\frac{1}{3}$, we have

$$
\Delta+\Lambda \approx 0.1831<1
$$

By Theorem 4.1, we know that problem (12) has at least one solution.

\section{Competing interests}

The author declares that he has no competing interests.

\section{Acknowledgements}

The author would like to express his thanks to the referees for their helpful suggestions. This work is partially supported by Shaoxing University (No. 20125009). 


\section{References}

1. Băleanu, D, Machado, JAT, Luo, ACJ: Fractional Dynamics and Control. Springer, Berlin (2012)

2. Sabatier, J, Agrawal, OP, Machado, JAT (eds.): Advances in Fractional Calculus: Theoretical Developments and Applications in Physics and Engineering. Springer, Dordrecht (2007)

3. Lakshmikantham, V, Leela, S, Vasundhara Devi, J: Theory of Fractional Dynamic Systems. Cambridge Scientific Publishers, Cambridge (2009)

4. Bai, ZB: On positive solutions of a nonlocal fractional boundary value problem. Nonlinear Anal. 72(2), 916-924 (2010)

5. Chen, AP, Tian, YS: Existence of three positive solutions to three-point boundary value problem of nonlinear fractional differential equation. Differ. Equ. Dyn. Syst. 18(3), 327-339 (2010)

6. Chen, $Y$, Tang, XH: Positive solutions of fractional differential equations at resonance on the half-line. Bound. Value Probl. 2012, 64 (2012)

7. Li, CF, Luo, XN, Zhou, Y: Existence of positive solutions of the boundary value problem for nonlinear fractional differential equations. Comput. Math. Appl. 59, 1363-1375 (2010)

8. Ahmad, B, Nieto, JJ: Anti-periodic fractional boundary value problems. Comput. Math. Appl. 62, 1150-1156 (2011)

9. Wang, GT, Ahmad, B, Zhang, LH: Impulsive anti-periodic boundary value problem for nonlinear differential equations of fractional order. Nonlinear Anal. 74(3), 792-804 (2011)

10. Wang, JR, LV, LL, Zhou, Y: Boundary value problems for fractional differential equations involving Caputo derivative in Banach spaces. J. Appl. Math. Comput. 38, 209-224 (2012)

11. Zhou, Y, Jiao, F: Nonlocal Cauchy problem for fractional evolution equations. Nonlinear Anal. 11, 4465-4475 (2010)

12. Zhang, S: Positive solutions for boundary-value problems of nonlinear fractional differential equations. Electron. J. Differ. Equ. 2006, 36 (2006)

13. Wang, JR, Zhou, Y, Feckan, M: On recent developments in the theory of boundary value problems for impulsive fractional differential equations. Comput. Math. Appl. 64, 3008-3020 (2012)

14. Wang, JR, Feckan, $M, Z$ Zhou, Y: On the new concept of solutions and existence results for impulsive fractional evolution equations. Dyn. Partial Differ. Equ. 8, 345-361 (2011)

15. Ahmad, B, Ntouyas, B, Alsaedi, S: New existence results for nonlinear fractional differential equations with three-point integral boundary conditions. Adv. Differ. Equ. 2011, Article ID 107384 (2011)

16. Ahmad, B: Nonlinear fractional differential equations with anti-periodic type fractional boundary conditions. Differ. Equ. Dyn. Syst. (2013). doi:10.1007/s12591-012-0154-2

17. Kilbas, AA, Srivastava, HM, Trujillo, JJ: Theory and Applications of Fractional Differential Equations. North-Holland Mathematics Studies, vol. 204. Elsevier, Amsterdam (2006)

18. Ahmad, B, Nieto, JJ, Alsaedi, A, Mohamad, N: On a new class of anti-periodic fractional boundary value problems. Abstr. Appl. Anal. 2013, Article ID 606454 (2013)

19. Hamani, S, Benchohra, M, Graef, JR: Existence results for boundary-value problems with nonlinear fractional differential inclusions and integral conditions. Electron. J. Differ. Equ. 2010, 20 (2010)

20. Cernea, A: Some remarks on a fractional differential inclusion with non-separated boundary conditions. Electron. J. Qual. Theory Differ. Equ. 2011, 45 (2011)

21. Chen, AP, Chen, Y: Existence of solutions to anti-periodic boundary value problem for nonlinear fractional differential equations. Differ. Equ. Dyn. Syst. 2011, Article ID 915689 (2011)

22. Wang, JR, Feckan, M, Zhou, Y: Ulam's type stability of impulsive ordinary differential equations. J. Math. Anal. Appl. 395, 258-264 (2012)

23. Wang, JR, Zhou, Y, Medved, M: Picard and weakly Picard operators technique for nonlinear differential equations in Banach spaces. J. Math. Anal. Appl. 389, 261-274 (2012)

24. Ahmad, B, Nieto, JJ: Riemann-Liouville fractional integro-differential equations with fractional nonlocal integral boundary conditions. Bound. Value Probl. 2011, 36 (2011)

25. Sudsutad, W, Tariboon, J: Boundary value problems for fractional differential equations with three-point fractional integral boundary conditions. Adv. Differ. Equ. 2012, 93 (2012)

26. Granas, A, Dugundji, J: Fixed Point Theory. Springer, New York (2003)

doi:10.1186/1687-1847-2013-257

Cite this article as: Fu: Existence results for fractional differential equations with three-point boundary conditions Advances in Difference Equations 2013 2013:257. 